\title{
Factores ambientales que modulan la fenología foliar de árboles del Bosque Atlántico
}

\author{
Débora di Francescantonio ${ }^{12,0}$; ; Mariana Villagra ${ }^{1,2}$; GuILlermo Goldstein ${ }^{3} \&$ \\ Paula I. Campanello ${ }^{1 / 4}$ \\ ${ }^{1}$ Laboratorio de Ecología Forestal y Ecofisiología, Instituto de Biología Subtropical, Universidad Nacional de Misiones, \\ CONICET. ${ }^{2}$ Asociación Civil Centro de Investigaciones del Bosque Atlántico (CeIBA). ${ }^{3}$ Laboratorio de Ecología Funcional, \\ Instituto de Ecología, Genética y Evolución de Buenos Aires, Facultad de Ciencias Exactas y Naturales, Universidad de Buenos \\ Aires, CONICET. ${ }^{4}$ Centro de Estudios Ambientales Integrados, Facultad de Ingeniería, Universidad Nacional de la Patagonia \\ San Juan Bosco, CONICET.
}

\begin{abstract}
Resumen. La fenología foliar responde de manera sensible a distintas señales ambientales. La coordinación entre las fases fenológicas y estas señales le permite a las especies ajustar el momento óptimo para expandir sus hojas y crecer, así como para evitar los posibles daños que causan las condiciones ambientales desfavorables. Por otro lado, en las especies deciduas, la altura de los árboles es otro factor clave, ya que los eventos de expansión y caída de las hojas de los individuos del dosel determinan la disponibilidad de luz para los de menor altura. El objetivo de nuestro trabajo fue conocer de qué manera los factores ambientales interactúan con la fenología foliar en especies arbóreas de diferente hábito foliar que coexisten en el extremo sur del Bosque Atlántico semideciduo. Para esto se monitoreó la fenología foliar de 10 especies arbóreas típicas del dosel del Bosque Atlántico semideciduo en la provincia de Misiones. En las especies deciduas y brevideciduas se identificaron patrones estacionales en las fases de expansión y caída de hojas, vinculados con las variaciones en las temperaturas y el fotoperíodo. En las siempreverdes, en cambio, no se encontró una asociación clara. Los árboles deciduos no dominantes adelantaron la expansión de hojas en comparación con los dominantes. Esto implicaría una estrategia para optimizar la captura de luz y la ganancia de carbono al inicio de la primavera extendiendo la estación de crecimiento. La fenología de las especies deciduas se acopló de forma estrecha a las variables ambientales, lo cual podría generar, en estas especies, mayor vulnerabilidad ante eventos extremos de estrés ambiental, como las bajas temperaturas y el déficit hídrico.
\end{abstract}

[Palabras clave: bosque subtropical, fotoperíodo, hábito foliar]

\begin{abstract}
Aвstract. Environmental factors modulating leaf phenology of trees in the Atlantic Forest. Leaf phenology responds sensitively to different environmental signals. Coordination between phenological phases and these signals allow species to adjust the optimal time for leaf expansion and growth, and to avoid possible damage caused by unfavorable environmental conditions. On the other hand, in deciduous species, tree height is another important factor, since the expansion and leafless events of canopy individuals determine the availability of light in lower heights. The objective of our work was to assess how environmental factors interact with leaf phenology in tree species of different leaf habits that coexist in the southern end of the semi-deciduous Atlantic Forest. For this purpose, the leaf phenology of 10 typical tree species of the canopy of the semi-deciduous Atlantic Forest in the province of Misiones was monitored. In deciduous and brevideciduous species, seasonal patterns were identified in the expansion and leafless phases linked to variations in temperature and photoperiod. In the evergreens, on the other hand, no clear association was found. The non-dominant deciduous trees advanced the expansion of leaves in comparison with the dominant ones. This would imply a strategy to optimize the capture of light and the gain of carbon at the beginning of the spring extending the season of growth. The phenology of deciduous species was closely coupled to environmental variables, which could generate, in these species, greater vulnerability to extreme events of environmental stress, such as low temperatures and water deficit.
\end{abstract}

[Keywords: subtropical forest, photoperiod, leaf habit]

\section{INTRODUCCIÓN}

La fenología describe los cambios en el tiempo de las diferentes etapas del ciclo de vida de los organismos (Aguirre et al. 2012; Borchert et al. 2014; Denny et al. 2014). Estos cambios son el resultado de la adaptación de las especies a las condiciones locales y a las señales ambientales que determinan el momento más eficiente para el crecimiento y la reproducción (van Schaik et al. 1993; Deslauriers et al. 2007; Forrest and MillerRushing 2010; Rossi and Bousquet 2014), los que, a su vez, están influenciados por la 
historia evolutiva de las especies (e.g., Davies et al. 2013; Panchen et al. 2014). En el caso de las plantas, sincronizar las etapas fenológicas con el período del año más favorable para la expansión de hojas y el crecimiento les permite satisfacer sus requerimientos metabólicos y fisiológicos, así como también evitar posibles daños causados por las condiciones ambientales desfavorables, como las bajas temperaturas invernales o la falta de agua (Badeck et al. 2004; Singh et al. 2017).

Las principales señales ambientales que regulan la fenología son la temperatura, las precipitaciones y el fotoperíodo, aunque la importancia de cada una varía entre diferentes ambientes. En los ecosistemas estacionales, como los bosques templados, el aumento de las temperaturas durante la primavera induce la producción sincrónica de hojas en la mayoría de las especies arbóreas, desencadenando así su crecimiento (Chuine and Régnière 2017). La disminución de la temperatura y del fotoperíodo, en cambio, promueven la senescencia y la caída de las hojas en los árboles (Delpierre et al. 2009; Borchert et al. 2005). En los bosques tropicales estacionalmente secos, en cambio, se considera que la variación de las precipitaciones es la principal señal ambiental que desencadena los eventos fenológicos, mientras que la variación sutil de la temperatura es una señal de menor importancia (Morellato et al. 2000, 2016; Borchert et al. 2002). En ambientes tropicales con baja estacionalidad climática, las señales invariantes año a año, como el fotoperíodo, son relevantes para definir el momento, la periodicidad y, en particular, la sincronía en los estados fenológicos (Borchert and Rivera 2001; Borchert et al. 2005).

La radiación solar también puede ser un factor determinante para la fenología de plantas arbóreas, en particular de aquellas que crecen bajo el dosel. Los eventos de expansión y caída de las hojas de los individuos de mayor altura son los principales determinantes de la disponibilidad de luz para las plantas de menor altura (Augspurger et al. 2005; Osada and Hiura 2019). Por lo tanto, las diferencias temporales entre la expansión y la maduración de las hojas podrían promover o dificultar la coexistencia de especies.

Las variaciones fenológicas y, en especial, los efectos del clima sobre la fenología de las hojas fueron muy estudiados en bosques templados y tropicales, pero en las regiones subtropicales estos estudios aún son escasos
(Cristiano et al. 2014; Zhang et al. 2016). En los bosques subtropicales de la Argentina, la temperatura y las precipitaciones determinan las diferencias de estacionalidad en los índices de productividad (Blundo et al. 2018). Sin embargo, los datos de análisis de sensores remotos en el Bosque Atlántico semideciduo no muestran una relación entre la dinámica fenológica y el clima (Blundo et al. 2018), posiblemente por la diversidad de especies siempreverdes y brevideciduas así como por el desarrollo de la vegetación del sotobosque durante el invierno, todo lo cual permite un crecimiento y un potencial fotosintético de la vegetación relativamente continuo a lo largo del año (Cristiano et al. 2014).

Considerando que la respuesta fenológica a las variables ambientales puede variar según el hábito foliar de las especies arbóreas, el objetivo de este trabajo fue conocer de qué manera los factores ambientales modulan la fenología en árboles con diferente hábito foliar que coexisten en el extremo sur del Bosque Atlántico semideciduo. Específicamente nos preguntamos: 1) Dada la escasa variabilidad estacional en las precipitaciones en el Bosque Atlántico semideciduo, ¿Son las variaciones en las temperaturas y en el fotoperíodo las señales que desencadenan los eventos fenológicos de las especies arbóreas?, 2) ¿Las especies con diferente hábito foliar responden de la misma manera a las variaciones ambientales?, y 3) ¿La posición de la copa de los árboles en el dosel influye en sus respuestas fenológicas? Para responder a estas preguntas, monitoreamos durante dos años la fenología foliar de 10 especies arbóreas del Bosque Atlántico semideciduo en la provincia de Misiones.

\section{Materiales y Métodos}

\section{Área de estudio}

El estudio se realizó al norte de la provincia de Misiones, dentro del Parque Nacional

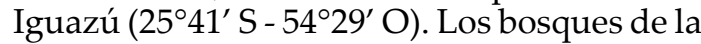
provincia de Misiones, o Selva Paranaense en la clasificación local (Oyazabal et al. 2018), se encuentran dentro de lo que se llama Bosque Atlántico semideciduo, según la clasificación original de Veloso et al. (1991) reeditada por el Instituto Brasileño de Geografía y Estadística (IBGE 2012). El Bosque Atlántico semideciduo se extiende sobre la provincia de Misiones, el sudeste de Paraguay y el sur de Brasil (OliveiraFilho and Fontes 2000; Oliveira-Filho et al. 2015). Estos bosques comparten especies con 
los bosques tropicales estacionalmente secos, así como también con los bosques húmedos de la costa atlántica en Brasil (Pennington et al. 2009; Werneck 2011; Banda-R et al. 2016). En el dosel de estos bosques coexisten especies arbóreas con diferentes hábitos foliares. El dosel está dominado por árboles del género Cordia L. (Boraginaceae), Ocotea Aubl. y Nectandra Rol. Ex Rottb. (Lauraceae), Parapiptadenia Brenan, Peltophorum (Bogel) Benth. (Fabaceae), Cedrela P. Browne, Trichilia P. Browne (Meliáceas), Plinia L., Eugenia L. y Myrciaria O. Berg. (Myrtaceae).

La precipitación anual media es de 2000 $\mathrm{mm}$. Las precipitaciones se distribuyen de forma homogénea a lo largo del año, aunque pueden ocurrir déficits hídricos de manera impredecible (Campanello et al. 2009). La temperatura media anual es de $21^{\circ} \mathrm{C}$, con medias mensuales de $25^{\circ} \mathrm{C}$ en enero y $15^{\circ} \mathrm{C}$ en julio, los meses más cálidos y más fríos del año, respectivamente. Las heladas (temperaturas menores a los $0{ }^{\circ} \mathrm{C}$ ) ocurren entre 2 a 9 días durante el año, entre los meses de junio y septiembre, dependiendo de la proximidad a los ríos y la altitud del sitio (Srur et al. 2007; Gatti et al. 2008).

\section{Hábito foliar}

Se estudiaron diez especies arbóreas representativas por su abundancia de los bosques de Misiones. La elección de las especies se realizó a partir de información bibliográfica (Zuloaga et al. 2008; Jardim Botânico do Rio de Janeiro 2018) y de observaciones previas en el área de estudio, de manera de representar los diferentes hábitos foliares presentes en el Bosque Atlántico semideciduo(siempreverdes, brevideciduas y deciduas) (Tabla 1). En total se seleccionaron y estudiaron 110 individuos (7 a 12 por especie). Todos los individuos fueron maduros, reproductivos y con un diámetro a la altura del pecho (DAP) entre 28 a 68 cm (Tabla 1). Se realizó un monitoreo durante dos años (agosto 2012 - julio 2014) observando la copa de cada árbol con binoculares la primera semana de cada mes. Para determinar la estacionalidad fenológica de las especies se cuantificó la intensidad de las diferentes fenofases en cada árbol (EF: hojas en expansión; HP: hojas en plenitud; $\mathrm{SH}$ : sin hojas). Para ello se empleó una escala de 0 a 4, representando el porcentaje de la copa en la que se presenta cada estado (Morellato et al. 2000; Borchert et al. 2002): 0=ausencia de la fenofase, $1=$ presencia de la fenofase con una magnitud entre $1-25 \%, 2=$ presencia de la fenofase con una magnitud entre $26-50 \%$, $3=$ presencia de la fenofase con una magnitud $51-75 \%$ y $4=$ presencia de la fenofase con una magnitud entre $76-100 \%$.

\section{Dominancia de los árboles}

Para evaluar si la posición de la copa de los árboles en el dosel influye en la fenología foliar, cada árbol se clasificó según la posición de su copa respecto a las copas de los árboles vecinos en árbol dominante y árbol no dominante. Los árboles dominantes fueron aquellos cuya copa superaba la altura de los árboles vecinos. Los árboles no dominantes incluyeron tanto a los árboles co-dominantes como a los suprimidos, los cuales tenían su

Tabla 1. Familias y especies de árboles estudiadas en el PN Iguazú, Argentina, hábito foliar (HF) (decidua [D], brevidecidua [B], siempreverde [S]); período en meses que los árboles están en la fenofase $\mathrm{SH}$ (media $\pm \mathrm{EE}$ ), diámetro a la altura del pecho (media $\pm E E$ ) y número de individuos clasificados como dominantes $(\mathrm{D})$ y no dominantes $(\mathrm{NoD})$ de cada especie.

Table 1. Family and species of trees studied in Iguazú National Park, Argentina, leaf habit (HF) (Deciduous [D], Brevideciduous [B], Evergreen [S]); leafless time (mean \pm SE), diameter at breast height (mean \pm SE) and number of individuals of each species classified as dominant (D) and non-dominant (ND).

\begin{tabular}{llccccc}
\hline Familia & Especie & HF & $\begin{array}{c}\text { Período SH } \\
(\mathrm{meses})\end{array}$ & $\begin{array}{c}\text { DAP } \\
(\mathrm{cm})\end{array}$ & D & NoD \\
\hline Malvaceae & Ceiba speciosa (A. St.-Hil., A. Juss. and Cambess.) Ravenna & $\mathrm{D}$ & $4.3 \pm 0.3$ & $41 \pm 13$ & 7 & 4 \\
Meliaceae & Cedrela fissilis Vell. & $\mathrm{D}$ & $5.5 \pm 0.4$ & $43 \pm 10$ & 5 & 6 \\
Boraginaceae & Cordia trichotoma (Vell.) Arráb. Ex Steud. & $\mathrm{D}$ & $5.9 \pm 0.2$ & $42 \pm 11$ & 7 & 5 \\
Meliaceae & Cabralea canjerana (Vell.) Mart. & $\mathrm{S}$ & $0.0 \pm 0.0$ & $45 \pm 8$ & 3 & 8 \\
Lauraceae & Ocotea diospyrifolia (Meisn.) Mez. & $\mathrm{S}$ & $0.0 \pm 0.0$ & $52 \pm 21$ & 5 & 6 \\
Sapotaceae & Chrysophyllum gonocarpum (Mart. and Eichler) Engl. & $\mathrm{S}$ & $0.0 \pm 0.0$ & $28 \pm 8$ & 3 & 9 \\
Rutaceae & Balfourodendron riedelianum (Engl.) Engl. & $\mathrm{B}$ & $1.1 \pm 0.3$ & $47 \pm 8$ & 10 & 0 \\
Fabaceae & Lonchocarpus muehlbergianus Hassl & $\mathrm{B}$ & $2.5 \pm 0.4$ & $38 \pm 6$ & 7 & 6 \\
Fabaceae & Parapiptadenia rigida (Benth.) Brenan & $\mathrm{B}$ & $2.6 \pm 0.2$ & $65 \pm 23$ & 4 & 3 \\
Fabaceae & Holocalyx balansae Micheli & $\mathrm{S}$ & $0.0 \pm 0.0$ & $68 \pm 8$ & 2 & 10 \\
\hline
\end{tabular}


copa a una altura igual o menor que los árboles vecinos, y en ambos casos la radiación solar no alcanzaba a toda la copa. Se consideraron árboles vecinos a aquellos que tenían su copa cercana de manera tal que pudieran afectar el acceso a la radiación solar.

\section{Variables ambientales}

Los registros de las precipitaciones y temperaturas del período estudiado fueron obtenidos de la estación meteorológica del Servicio Meteorológico Nacional ubicada en el Aeropuerto de Puerto Iguazú (a una distancia de $\sim 8 \mathrm{~km}$ del sitio de estudio). La cantidad de horas luz para el sitio de estudio se obtuvo empleando el paquete geosphere (Hijmans 2016) en el programa R a partir de las coordenadas geográficas del área de estudio (R Core Team 2016).

\section{Análisis de datos}

La intensidad (0-4) de las fenofases (EF, HP y $\mathrm{SH}$ ) obtenida mensualmente en cada uno de los 110 individuos de las 10 especies estudiadas se utilizó para obtener la frecuencia mensual de las diferentes fenofases por especie. Se evaluó la estacionalidad de estas fenofases empleando estadística circular (Morellato et al. 2010), considerando el período anual como un ciclo. Para ello, los meses del año fueron convertidos a ángulos asignando $30^{\circ}$ a cada mes (septiembre, $0^{\circ}$; octubre, $30^{\circ}$; noviembre, $60^{\circ}$, etc.). Se generaron histogramas circulares de frecuencias (Pewsey et al. 2013) de las diferentes fenofases, por especie y año evaluado. En cada histograma se calculó el vector medio (dirección, es decir el ángulo medio, e intensidad o módulo del vector) (Morellato et al. 2010), que indica la tendencia central de los datos (momento del año en que se observa una determinada fenofase). La estacionalidad de cada fenofase, concentración de los datos alrededor del ángulo medio, está dada por el parámetro $r$. Este parámetro toma valores de cero (cuando existe tanta dispersión que el ángulo medio no puede ser detectado) a uno (cuando todos los datos están concentrados en la dirección del ángulo medio) (Pewsey et al. 2013). Para determinar su significancia se utilizó la prueba de Rayleigh (Zar 1999). Luego, para evaluar si existen diferencias entre los dos años de estudio en la estacionalidad de las fenofases de cada especie se utilizó la prueba de Watson-Williams (F) (Morellato et al. 2010). Esta prueba permite comparar los ángulos medios entre dos series de datos; en este caso se comparan las series correspondientes a las fenofases (EF, HP y SH) del primer año con el segundo. Los análisis se realizaron empleando el paquete circular (Lund et al. 2017) en el programa R (R Core Team 2016).

Para estudiar las relaciones entre las diferentes fenofases foliares de cada especie y las variables ambientales (precipitación mensual, temperatura y fotoperíodo) se realizaron correlaciones cruzadas. Se emplearon, para cada especie por separado, los promedios mensuales (la unidad muestral es el individuo, $\mathrm{N}=7-12$ según la especie) de cada fenofase y la temperatura máxima, media y mínima, precipitaciones y fotoperíodo, medidos durante los dos años consecutivos (serie temporal de 24 meses). Las correlaciones cruzadas permiten determinar los desfasajes temporales entre dos series de datos que fueron medidas en el mismo intervalo de tiempo. El análisis arroja dos valores, el valor de máxima correlación $\left(R_{\text {máx }}\right)$ que puede ser positivo o negativo según la correlación sea directa o indirecta, y el valor de desfasaje, también positivo o negativo, según sean retrasos o adelantamientos de la primera serie en relación a la segunda. En este trabajo la unidad de los desfasajes fue un mes debido a la frecuencia del muestreo.

El análisis de correlaciones cruzadas también fue empleado para evaluar cómo la posición de la copa de los árboles en el dosel influye en su fenología foliar. Dado que el número de individuos presentes en cada categoría de dominancia (dominante/no-dominante) era muy bajo para algunas especies (Tabla 1), los árboles fueron agrupados según su hábito foliar. La comparación se realizó entre árboles dominantes y no dominantes, separados según su hábito foliar y fenofase, es decir, en total se realizaron 9 correlaciones. Las series temporales para cada categoría de dominancia empleadas en este análisis se construyeron a partir de los valores medios mensuales de cada fenofase por especie, luego promediados según su hábito foliar. Los análisis se realizaron con el software InfoStat versión 2017 (Di Rienzo et al. 2017).

\section{Resultados}

\section{Hábito foliar y variables ambientales}

Las especies deciduas permanecieron con sus copas sin hojas durante 3 meses o más, las brevideciduas tuvieron un período sin hojas menor a 3 meses y las siempreverdes 

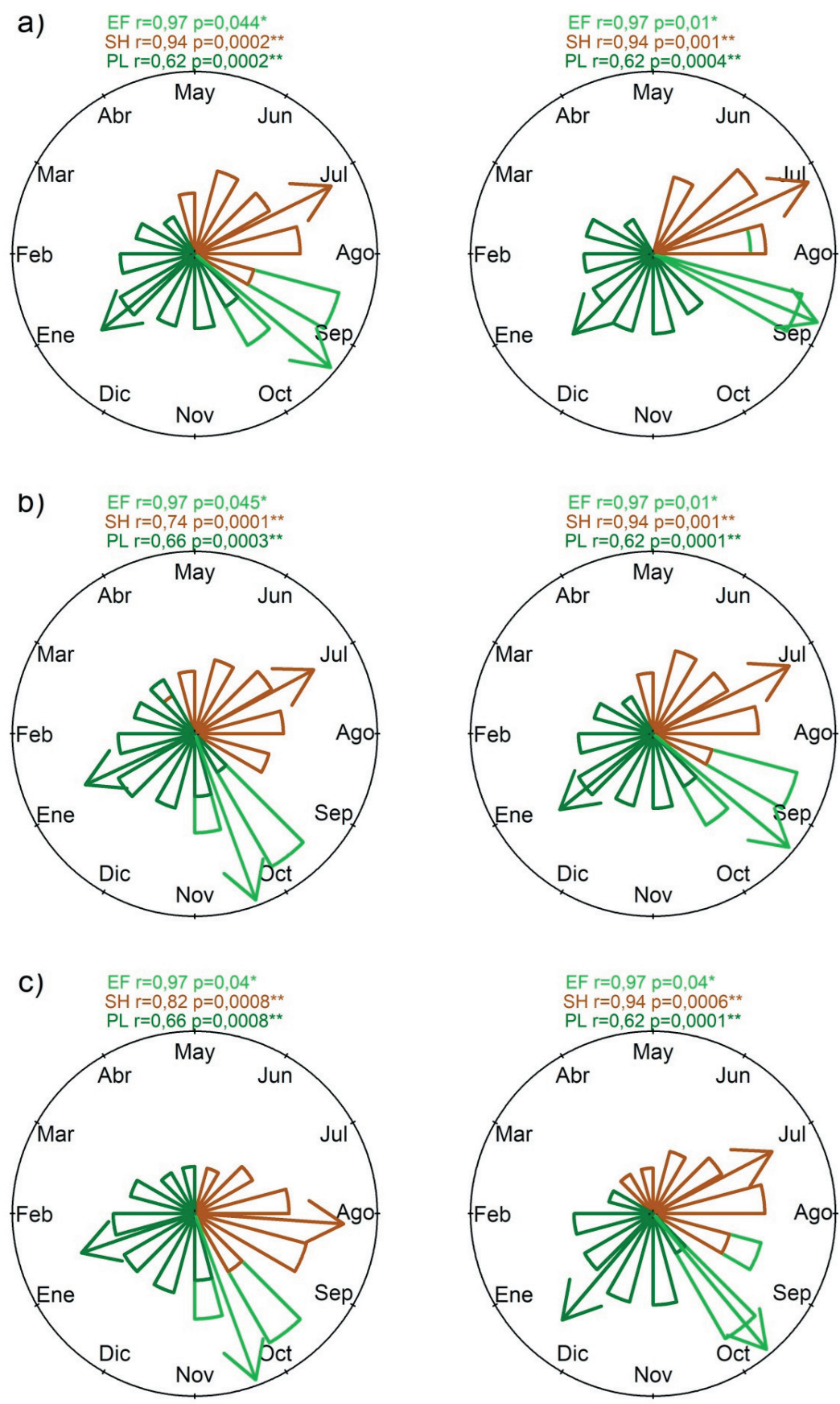

Figura 1. Distribución mensual de la frecuencia e intensidad de las fenofases foliares: expansión foliar (EF), hojas en plenitud (PL) y sin hojas (SH) para especies deciduas a) C. fissilis, b) C. speciosa y c) C. trichotoma durante dos años consecutivos, desde agosto 2012 a julio 2014, en el PN Iguazú, Argentina. Izquierda: primer año; derecha: segundo año. $r=$ concentración de las fenofases alrededor del ángulo medio (flechas), $P=$ significancia del ángulo medio dada por la prueba de Rayleigh, ${ }^{*} P<0.05,{ }^{* *} P<0.01,{ }^{* * *} P<0.001$. Los colores indican las fenofases (EF: verde claro, PL: verde oscuro, SH: marrón).

Figure 1. Monthly distribution of the frequency and intensity of phenophases: foliar expansion (EF), mature leaves $(\mathrm{PL})$, and leafless (SH) for deciduous species a) C. fissilis, b) C. speciosa and c) C. trichotoma, during two consecutive years, from August 2012 to July 2014, in the Iguazú NP, Argentina. Left: first year; right: second year. r=concentration of phenophases around mean angle (arrows), $P=$ significance of mean angle given by Rayleigh test. ${ }^{*} P<0.05,{ }^{* *} P<0.01$, ${ }_{* * *} P<0.001$. Colors indicate phenophases (EF: light green, PL: dark green, SH: brown). 
no presentaron en ningún momento sus copas sin hojas (Tabla 1). En las especies deciduas, el ángulo medio para la fase sin hojas en general corresponde al mes de julio y la concentración alrededor del ángulo medio $(r)$ fue mayor a 0.7 (Figura 1). Además, en estas especies se encontraron correlaciones significativas entre el aumento de la fase sin hojas y la disminución de las temperaturas y de la cantidad de horas de luz, tanto para el mismo mes (desfasaje $=0$ ) como para el mes anterior (desfasaje=1) (Tabla S2). Las fases de expansión foliar y de plenitud presentaron el ángulo medio entre los meses de septiembre-octubre y diciembre-enero, respectivamente, con un alto grado de estacionalidad ( $r>0.9$ para la expansión foliar y $r>0.6$ para la plenitud foliar) (Figura 1). La fase de expansión foliar de $C$. speciosa se correlacionó positivamente con las temperaturas medias (desfasaje $=-2$, adelanto de la fenofase respecto a la serie temporal de temperaturas medias) y las precipitaciones (desfasaje $=4$, retraso de la fase en relación a las precipitaciones), y negativamente con las temperaturas mínimas (desfasaje=2). En $C$. fissilis, esta fase se correlacionó negativamente con las temperaturas mínimas (desfasaje=1) y con el fotoperíodo (desfasaje=1), mientras que en $C$. trichotoma se asoció positivamente con las temperaturas máximas (desfasaje= -4) y las temperaturas medias (desfasaje= -2), y negativamente con las temperaturas mínimas (desfasaje=2) (Tabla S2, Figura 4). La fenofase de plenitud de las hojas se asoció positivamente con un aumento en las temperaturas y en la cantidad de horas de luz diarias (Figura 1, Tabla S2).

En las especies brevideciduas, el ángulo medio para la fase sin hojas varió entre las diferentes especies (Figura 2). En el caso de $B$. riedelianum no se encontró un vector medio significativo (indicando baja estacionalidad o intervalos de tiempo menores a un mes). La caída de hojas en estas especies brevideciduas se asoció significativamente con la disminución de las temperaturas y de la cantidad de horas de luz diarias, pero los desfasajes fueron diferentes entre las especies, en L. muehlbergianus fue de tres meses mientras que en $B$. riedelianum y $P$. rigida resultó de un mes (Tabla S3). En las tres especies la fase sin hojas estuvo retrasada en relación a las variables ambientales (desfasajes positivos). En este grupo de especies, la fase de expansión de hojas, en general, no presentó un vector medio significativo (Figura 2), sin embargo se registraron correlaciones significativas con las variables ambientales. En L. muehlbergianus se asoció positivamente con las temperaturas medias, mínimas y con el fotoperíodo (con desfasajes $=0$ en los tres casos), mientras que para $B$. riedelianum y $P$. rigida se asoció positivamente con las precipitaciones (desfasaje $=4$ ) (Tabla S3, Figura 4).

En las especies siempreverdes, los vectores medios para las tres fenofases estudiadas (i.e., expansión foliar, hojas en plenitud y sin hojas) no resultaron significativos (Figura 3). Este grupo de especies presentó, en comparación con las especies deciduas y brevideciduas, muy pocas correlaciones entre las fenofases y las variables ambientales estudiadas, y estas correlaciones fueron variables entre las especies (Tabla S4). Cabralea canjerana no presentó correlaciones significativas entre ninguna de las fenofases y las variables ambientales evaluadas. En el caso de $O$. diospyrifolia se observaron correlaciones negativas entre la expansión foliar y las temperaturas máximas (desfasaje $=4$ ) y el fotoperíodo (desfasaje=4), mientras que para

Tabla 2. Desfasajes temporales entre árboles dominantes y no dominantes para cada hábito foliar (deciduo, brevideciduo y siempreverde) y fase (hojas en expansión [EF]; hojas en plenitud [PL] y sin hojas [SH]). Los desfasajes indican el número de meses en que se adelantan (desfasaje negativo) o retrasan (desfasajes positivos) las fenofases foliares de los árboles no dominantes en relación con los dominantes para las especies estudiadas durante dos años consecutivos (agosto 2012 a julio 2014) en el PN Iguazú, Argentina. Se muestra el coeficiente de máxima correlación $\left(\mathrm{R}_{\text {máx }}\right)$ y el valor del estadístico $t$ y su nivel de significancia $\left({ }^{*} P<0.05 ;{ }^{* *} P<0.001\right)$. ns: diferencias no significativas.

Table 2. Temporal lags between dominant and no-dominant trees for each leaf habit (deciduous, brevideciduos, and evergreen) and phenophases (foliar expansion [EF]; mature leaves [PL] and leafless [SH]). Lags indicate the number of months in which foliar phenophases begin earlier (negative lag) or later (positive lag) for non-dominant trees in relation to dominant ones for the species studied during two consecutive years (August 2012 to July 2014) in the Iguazú National Park. Maximum correlation coefficient $\left(\mathrm{R}_{\mathrm{mix}}\right)$ and $t$ statistic value and significant level $\left({ }^{*} P<0.05 ;{ }^{* *} P<0.001\right)$ are shown. ns: no significant difference.

\begin{tabular}{lcccc}
\hline Hábito foliar & Fenofase & $\begin{array}{c}\text { Desfasaje } \\
\text { (meses) }\end{array}$ & $\mathrm{R}_{\text {máx }}$ & $t$ \\
\hline Deciduo & EF & 1 & 0.82 & $2.8^{*}$ \\
& PL & 0 & 0.9 & $3.44^{* *}$ \\
Brevideciduo & SH & 0 & 0.9 & $3.43^{* *}$ \\
& EF & -2 & 0.63 & $2.12^{*}$ \\
& PL & 0 & 0.72 & $2.07^{*}$ \\
Siempreverde & SH & 0 & 0.82 & $2.85^{*}$ \\
& EF & 0 & 0.82 & $2.87^{* *}$ \\
& PL & 0 & 0.85 & $2.95^{* *}$ \\
& SH & 0 & 0.6 & $2.97^{*}$ \\
\hline
\end{tabular}



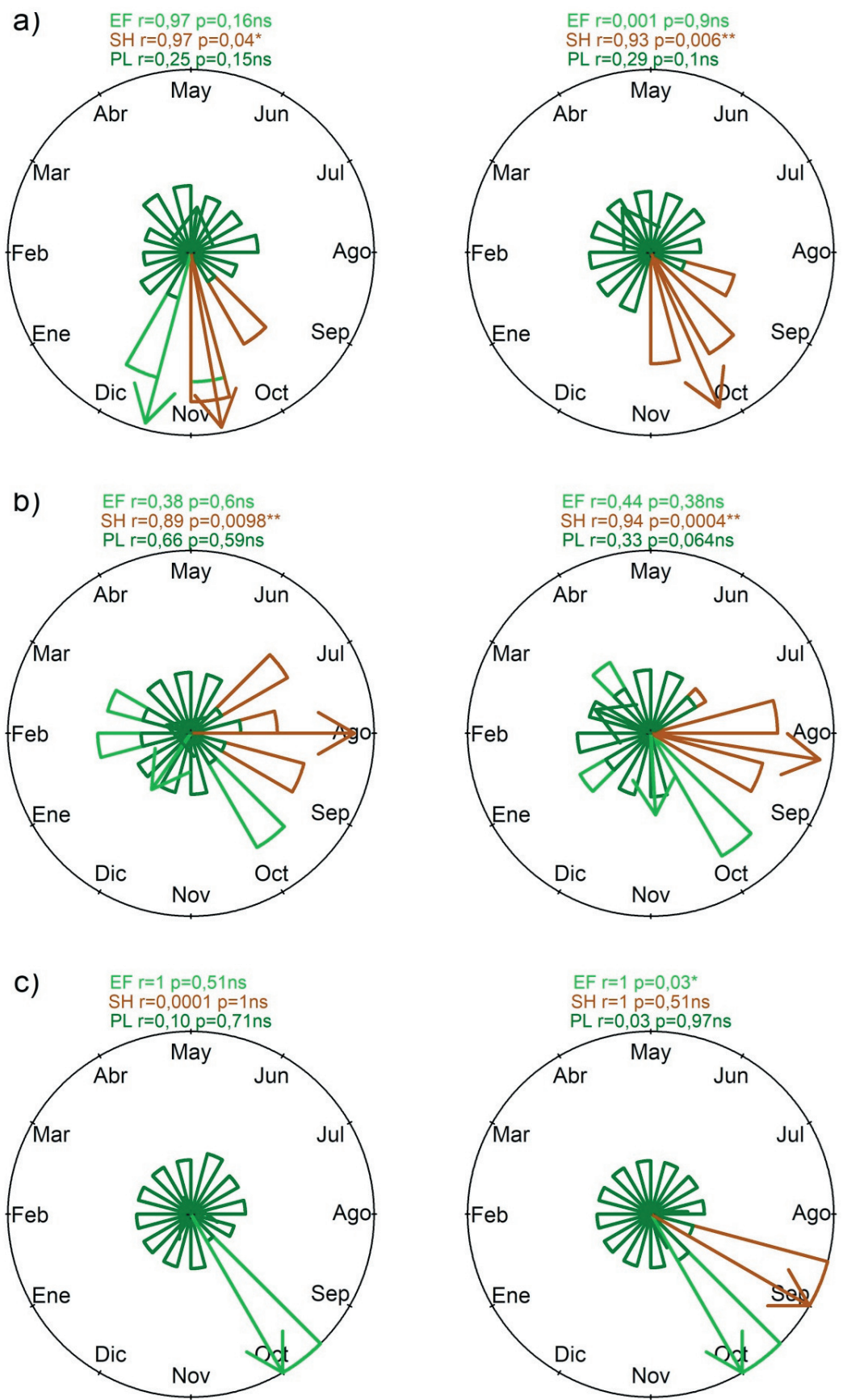

Figura 2. Distribución mensual de la frecuencia e intensidad de las fenofases foliares de especies brevideciduas: expansión foliar (EF), hojas en plenitud (PL) y sin hojas (SH) para especies brevidedeciduas a) L. muehlbergianus, b) $P$. rigida y c) B. riedelianum durante dos años consecutivos, desde agosto 2012 a julio 2014, en el PN Iguazú. Izquierda: primer año; derecha: segundo año. $r=$ concentración de las fenofases alrededor del ángulo medio (flechas), $P=$ significancia del ángulo medio dada por la prueba de Rayleigh, ${ }^{*} P<0.05,{ }^{* *} P<0.01,{ }^{* * *} P<0.001$. Los colores indican las fenofases (EF: verde claro, PL: verde oscuro, SH: marrón).

Figure 2. Monthly distribution of the frequency and intensity of phenophases: foliar expansion (EF), mature leaves $(\mathrm{PL})$, and leafless $(\mathrm{SH})$ for brevideciduous species a) L. muehlbergianus, b) P. rigida and c) B. riedelianum, during two consecutive years, from August 2012 to July 2014, in the Iguazú NP, Argentina. Left: first year; right: second year. $r=$ concentration of phenophases around mean angle (arrows), $P=$ significance of mean angle given by Rayleigh test. ${ }^{*} P<0.05,{ }^{* *} P<0.01,{ }^{* * *} P<0.001$. Colors indicate phenophases (EF: light green, PL: dark green, SH: brown). 

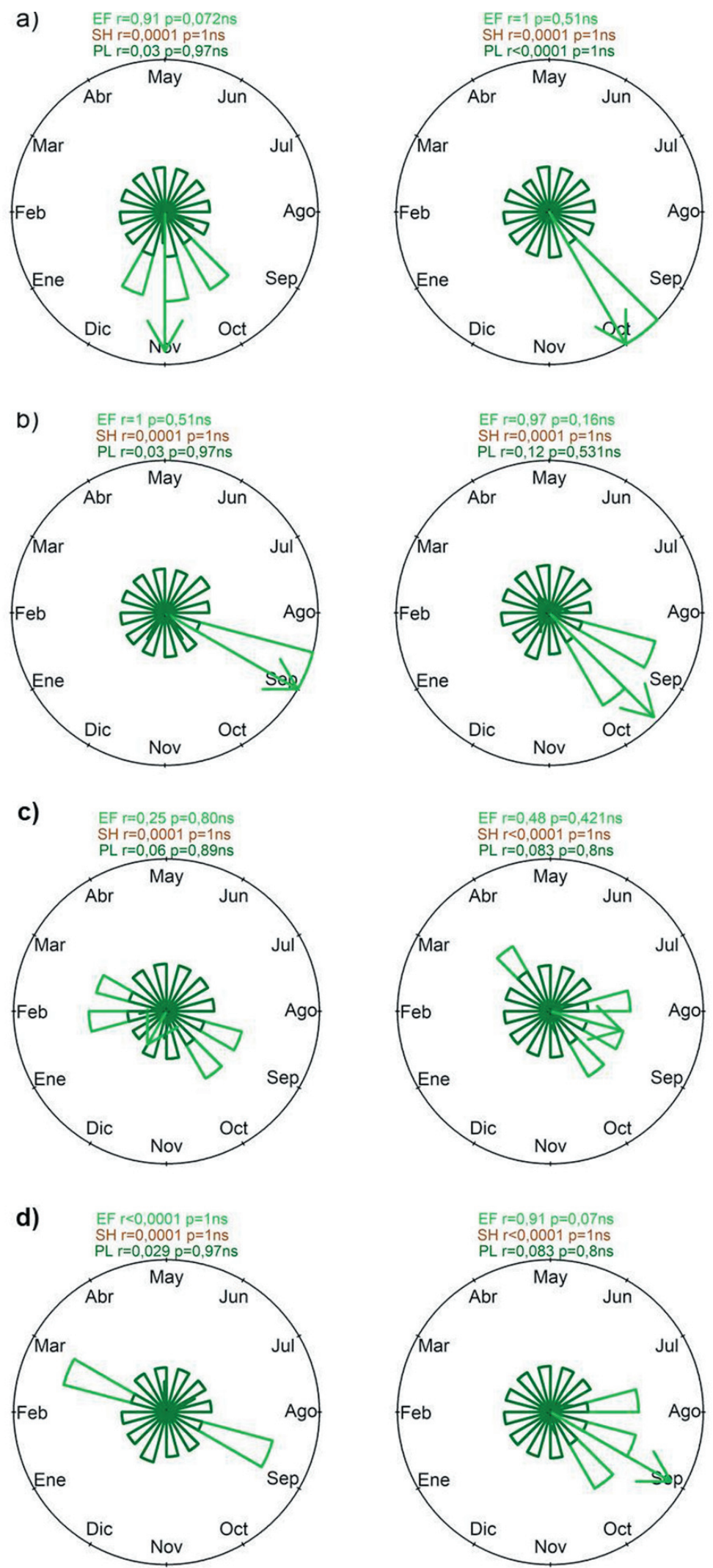

Figura 3. Distribución de la frecuencia e intensidad de las fenofases foliares de especies siempreverdes: expansión foliar (EF), hojas en plenitud (PL) y sin hojas (SH) mensual para especies siempreverdes a) C. gonocarpum, b) O. diospyrifolia , c) C. canjerana, d) $H$. balansae durante dos años consecutivos desde agosto 2012 a julio 2014. Izquierda: primer año, derecha: segundo año. $\mathrm{r}=$ concentración de las fenofases alrededor del ángulo medio (flechas), p=significancia del ángulo medio dada por la prueba de Raleigh, ${ }^{*} p<0,05, \quad{ }^{* *} p<0,01$, ${ }_{* * *} \mathrm{p}<0,001$.

Figure 3. Monthly distribution of the frequency and intensity of phenophases: foliar expansion (EF), mature leaves (PL), and leafless (SH) for evergreen species a) C. gonocarpum, b) O. diospyrifolia c) C. canjerana, d) H. balansae during two consecutive years from August 2012 to July 2014. Left: first year, right: second year. $r=$ concentration of phenophases around mean angle (arrows), $p=$ meaning of mean angle given by Raleigh test. ${ }^{*} \mathrm{p}<0.05$, ${ }^{* *} \mathrm{p}<0.01,{ }^{* * *} \mathrm{p}<0.001$. 

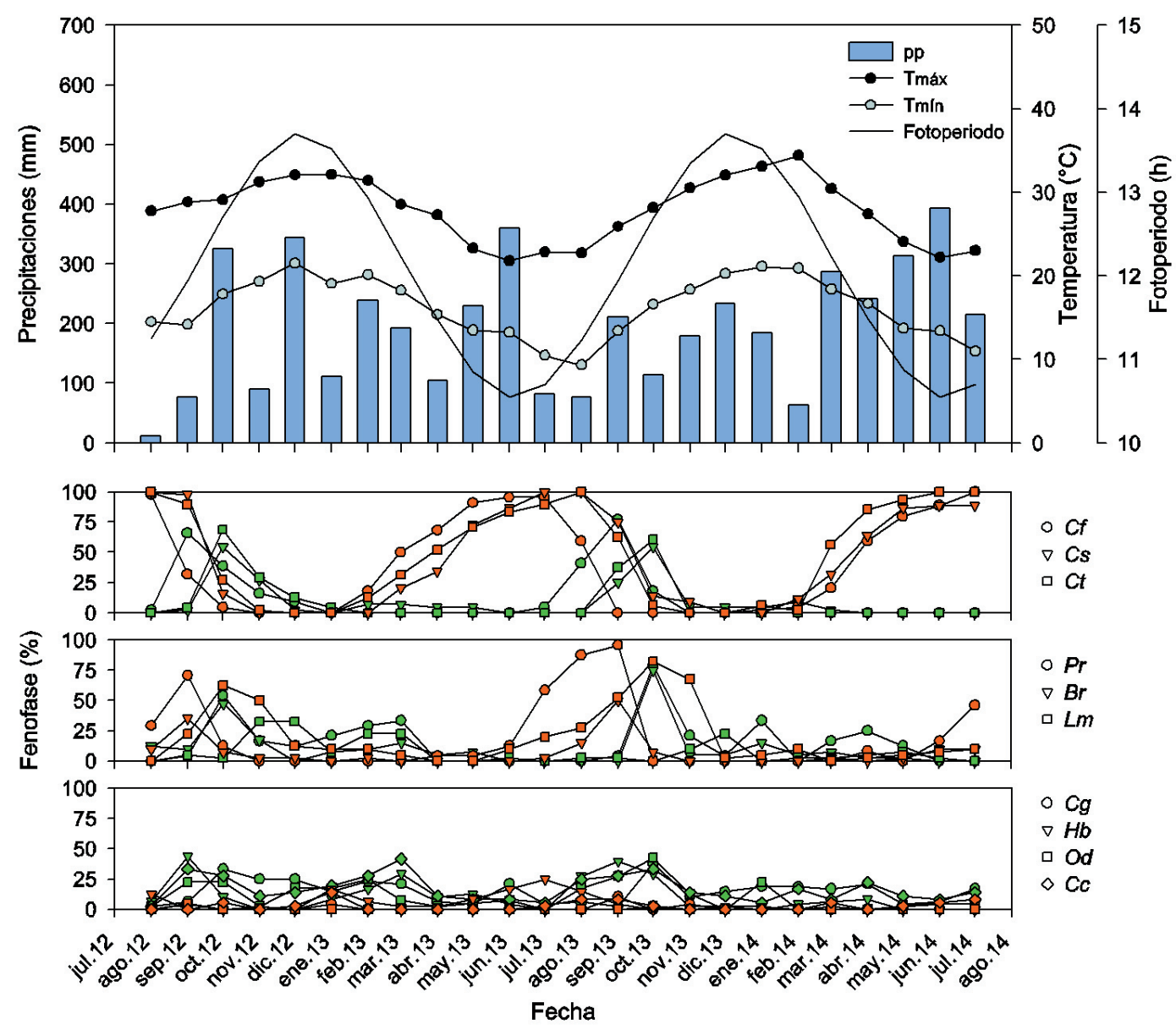

Figura 4. Panel superior: precipitaciones $(\mathrm{mm})$, temperatura máxima $\left(\mathrm{T}_{\text {max }}\right)$, media $\left(\mathrm{T}_{\text {med }}\right)$ y mínima $\left(\mathrm{T}_{\text {min }}\right)$ mensuales, $\mathrm{y}$ fotoperíodo para el sitio de estudio en el período agosto 2012- julio 2014. Panel inferior: fenofases foliares en el mismo período de las especies deciduas, brevideciduas y siempreverdes en el PN Iguazú. Sólo se indican las fases de expansión de hojas (verde) y sin hojas (naranja). Especies deciduas: C. fissilis (Cf), C. speciosa (Cs) y C. trichotoma (Ct). Especies brevideciduas: P. rigida $(\mathrm{Pr})$, B. riedelianum $(\mathrm{Br})$ y L. muehlbergianus $(\mathrm{Lm})$. Especies siempreverdes: C. gonocarpum $(\mathrm{Cg})$, H. balansae $(\mathrm{Hb})$, O. diospyrifolia $(\mathrm{Od})$ y C. canjerana $(\mathrm{Cc})$.

Figure 4. Upper panel: precipitation $(\mathrm{mm})$, maximum temperature $\left(\mathrm{T}_{\text {max }}\right)$, mean $\left(\mathrm{T}_{\text {med }}\right)$ and minimum $\left(\mathrm{T}_{\min }\right)$, and photoperiod for the study site between August 2012 and July 2014. Bottom panel: leaf phenology for deciduous, brevideciduous, and evergreen species for the same period in the Iguazú NP. Only leaf expansion (green) and leafless phases (orange) are indicated. Deciduous species: C. fissilis (Cf), C. speciosa (Cs) and C. trichotoma (Ct). Brevideciduous species: $P$. rigida $(\mathrm{Pr})$, B. riedelianum $(\mathrm{Br})$ and L. muehlbergianus $(\mathrm{Lm})$. Evergreen species: C. gonocarpum $(\mathrm{Cg})$, H. balansae $(\mathrm{Hb})$, O. diospyrifolia $(\mathrm{Od})$ and C. canjerana $(\mathrm{Cc})$.

C. gonocarpum las correlaciones entre la fase de expansión de hojas y las temperaturas medias, mínimas y el fotoperíodo fueron positivas (desfasaje=0) (Tabla S4, Figura 4).

\section{Fenología de árboles dominantes y no dominantes}

La fase de expansión foliar a lo largo del año resultó diferente entre los árboles dominantes y los no dominantes para las especies deciduas y brevideciduas. En las especies deciduas se registró el adelantamiento de un mes en la sali da de las hojas en los árboles no dominantes (desfasaje=1), mientras que en las brevideci duas un retraso (desfasaje=-2). En las especies siempreverdes no se registraron diferencias significativas entre los árboles dominantes y no dominantes (Tabla 2, Figura S1).

\section{Discusión}

Las especies deciduas y brevideciduas presentaron una fenología más asociada a las variaciones ambientales, principalmente en la fases de expansión foliar y de caída de hojas, en comparación con las siempreverdes (Figura 4). Estos resultados son consistentes con los encontrados en otras áreas del subtrópico 
(Morellato et al. 2000; Marques et al. 2004; Bianchini et al. 2015) en donde la estacionalidad está dada por cambios en las temperaturas y en el fotoperíodo, y no por las precipitaciones. En parte, estos resultados también se reflejan en las observaciones de Blundo et al. (2018) a escala satelital, en las que estos bosques no presentan una marcada estacionalidad, probablemente por la relación en el número de especies deciduas y siempreverdes.

Entre las especies estudiadas, C. canjerana, considerada como especie siempreverde según la bibliografía de la región (Zuloaga et al. 2008; Jardim Botânico do Rio de Janeiro 2018), fue clasificada como brevidecidua en un estudio reciente realizado al norte del Bosque Atlántico (Shimamoto et al. 2016). Las especies L. muehlbergianus y B. riedelianum, consideradas brevideciduas en este trabajo, fueron catalogadas como deciduas hacia el sur de sus distribuciones en el sur del Bosque Atlántico en el estado de Rio Grande do Sul (Franco 2008).

No es raro que algunas especies cambien su hábito foliar a escala local o regional dependiendo de la duración de la estación favorable. Según Kikuzawa et al. (2013), esta plasticidad en el patrón fenológico podría ayudar a entender la evolución latitudinal de las plantas. Desde un punto de vista filogenético y funcional, el hábito brevideciduo sería una variante del hábito deciduo más que un verdadero hábito siempreverde (Kikuzawa and Lechowicz 2011). En los extremos de las distribuciones geográficas de especies de bosques tropicales estacionalmente secos, donde las sequías no son tan prolongadas (mayores precipitaciones y distribuidas homogéneamente), especies típicamente deciduas pueden comportarse como brevideciduas (Borchert et al. 2005; Kikuzawa and Lechowicz 2011).

Los cambios fenológicos en nueve de las diez especies arbóreas estudiadas del Bosque Atlántico semideciduo respondieron a las variaciones en la temperatura, siete especies a variaciones en el fotoperíodo y cuatro a variaciones en las precipitaciones. La fase sin hojas presentó asociaciones con las disminuciones de la temperatura y del fotoperíodo con desfasajes temporales máximos de un mes. Si bien fue posible identificar períodos asociados a la expansión foliar (septiembre-noviembre) y a la caída de hojas (abril-septiembre) para el conjunto de las especies deciduas y brevideciduas, las repuestas a las variaciones ambientales resultaron especie-específicas. Se encontraron adelantamientos de la fase de expansión de hojas en relación a las temperaturas en algunas especies. En C. speciosa esta fase ocurrió dos meses antes del aumento de las temperaturas medias, mientras que en $C$. trichotoma se observó cuatro meses antes de los aumentos de las temperaturas máximas. Estos desfasajes indicarían que estas especies evitan las altas temperaturas para expandir las hojas. La sensibilidad que presentan las yemas y el desarrollo en sí de las hojas a la temperatura resaltan la importancia que tiene el momento en que se desencadena esta fenofase, así como su valor adaptativo, ya que asegura la maximización de la estación de crecimiento al mismo tiempo que minimiza el peligro de daño por heladas (Kramer et al. 2010).

Los árboles deciduos no dominantes estudiados en este trabajo adelantaron la expansión de hojas en comparación a los dominantes. Este comportamiento ya fue registrado en otras especies deciduas de bosques estacionales (Augspurger et al. 2005; Vitasse 2013). Esto sugiere que tanto la expansión, como la maduración temprana de las hojas son estrategias que permiten que los árboles más pequeños mejoren la captura de luz y la ganancia de carbono a principios de la primavera, aun a riesgo de mayores probabilidades de daños por heladas (Vitasse et al. 2014). En el caso de las especies brevideciduas, se registró un retraso en la fase de expansión foliar de los árboles no dominantes, aunque es posible que estos resultados presenten un sesgo generado por la especie $B$. riedelianum, para la que sólo estudiamos árboles dominantes (Tabla 1).

Las especies deciduas y brevideciduas en nuestro estudio presentaron su fenología estrechamente acoplada a las variables ambientales. Según varios autores, estos acoplamientos resultan en mayor vulnerabilidad de las especies ante el cambio climático, como, por ejemplo, ante pequeños cambios en la estacionalidad de la temperatura o en el patrón de precipitaciones (Hódar et al. 2004; Lasky et al. 2016; Fu et al. 2019). Si bien las especies en general presentan cierta plasticidad en la respuesta a las variaciones ambientales, pueden generarse desacoples en diferentes niveles tróficos alterando interacciones mutualistas (entre plantas y polinizadores o dispersores de semillas) y antagonistas (entre plantas y herbívoros) (Morellato et al. 2016) y, en última instancia, el 
fitness de las especies y su distribución espacial (Chuine and Beaubien 2001).

Además de los posibles impactos relacionados al cambio climático, es necesario considerar el efecto del aumento en la presión de uso sobre los ecosistemas forestales. El uso de los bosques puede afectar el funcionamiento de los ecosistemas a través de cambios en la estructura y en la composición de las especies (Campanello et al. 2007a, 2007b), y modificar asimismo la frecuencia de los diferentes hábitos fenológicos (Blundo et al. 2018). Debido a esto, resulta importante desarrollar estudios a largo plazo que permitan comprender cómo la fenología se ajusta a las variables ambientales en las diferentes especies, así como también la relación que presentan los patrones fenológicos con rasgos funcionales que determinan la economía de carbono, el uso del agua y el ciclado de nutrientes. Por ejemplo, un subconjunto de las especies estudiadas es capaz de usar gran parte de sus reservorios de agua ubicados en los troncos principales y ramas durante la fase sin hojas, probablemente para desarrollar hojas nuevas durante la expansión foliar (di Francescantonio et al. 2018). Esto les aseguraría cierta independencia fenológica con respecto a las precipitaciones. Sin embargo, la capacidad de carga de estos reservorios depende no solo de las características morfo-fisiológicas de los tejidos sino también de la disponibilidad de agua en el suelo (Köcher et al. 2013), por lo tanto, una modificación en el patrón de precipitaciones podría afectar la capacidad de almacenamiento y el uso de estos reservorios de agua. Conocer los cambios fenológicos es fundamental para mejorar las predicciones de modelos de flujo de carbono y agua en esta región y a escala global (Seki et al. 2015) así como también para evaluar las posibles consecuencias del cambio climático.

Agradecimientos. Este trabajo fue financiado parcialmente por la ANPCyT (PICT 20122084 y PICT PITEC-Aglomerado Productivo Forestal Misiones y Norte de Corrientes). Agradecemos a la Administración de Parques Nacionales (APN) por permitirnos realizar este trabajo en el Parque Nacional Iguazú, a Oscar Lezcano, Laureano Oliva Carrasco y Yamil Di Blanco por la ayuda en los trabajos de campo.

\section{REFERENCIAS}

Aguirre, L., E. Anderson, B. Gunnar, S. Herzog, and P. Jørgensen. 2012. Fenología y relaciones ecológicas interespecíficas de la biota andina frente al cambio climático. Cambio Climático y Biodiversidad en los Andes Tropicales. Pp. 428.

Augspurger, C. K., J. M. Cheeseman, and C. F. Salk. 2005. Light gains and physiological capacity of understorey woody plants during phenological avoidance of canopy shade. Functional Ecology 19:537-546. https://doi.org/10.1111/j.13652435.2005.01027.x.

Badeck, F. W., A. Bondeau, K. Böttcher, D. Doktor, W. Lucht, J. Schaber, and S. Sitch. 2004. Responses of spring phenology to climate change. New Phytologist 162:295-309. https://doi.org/10.1111/j.1469-8137.2004.01059.x.

Banda-R, K., A. Delgado-Salinas, K. G. Dexter, R. Linares-Palomino, Oliveira-Filho, P. Ary, M. Pullan, C. Quintana, and R. Ricarda. 2016. Plant diversity patterns in neotropical dry forests and their conservation implications. Science 353:1383-1388. https://doi.org/10.1126/science.aaf5080.

Bianchini, E., J. M. Emmerick, A. V. L. Messetti, and J. A. Pimenta. 2015. Phenology of two Ficus species in seasonal semi-deciduous forest in Southern Brazil. Brazilian Journal of Biology 75:206-214. https://doi.org/10.1590/15196984.10614.

Blundo, C., N. I. Gasparri, A. Malizia, M. Clark, G. Gatti, P. I. Campanello, H. R. Grau, L. Paolini, L. R. Malizia, S. E. Chediack, P. Macdonagh, and G. Goldstein. 2018. Relationships among phenology, climate and biomass across subtropical forests in Argentina. Journal of Tropical Ecology 34:93-107. https://doi.org/10.1017/S026646741800010X.

Borchert, R., Z. Calle, A. H. Strahler, A. Baertschi, R. E. Magill, J. Broadhead, J. Kamau, J. Njoroge, and C. Muthuri. 2014. Insolation and photoperiodic control of tree development near the equator. New Phytologist 205:7-13. https: //doi.org/10.1111/nph.12981.

Borchert, R., and G. Rivera. 2001. Photoperiodic control of seasonal development and dormancy in tropical stemsucculent trees. Tree Physiology 21:213-221. https://doi.org/10.1093/treephys/21.4.213.

Borchert, R., G. Rivera, and W. Hagnauer. 2002. Modification of Vegetative Phenology in a Tropical Semi-deciduous Forest by Abnormal Drought and Rain1. Biotropica 34:27-39. https://doi.org/10.1646/0006-3606(2002)034[0027: MOVPIA]2.0.CO;2. https://doi.org/10.1111/j.1744-7429.2002.tb00239.x.

Borchert, R., K. Robertson, M. D. Schwartz, and G. Williams-Linera. 2005. Phenology of temperate trees in tropical climates. International Journal of Biometeorology 50:57-65. https://doi.org/10.1007/s00484-005-0261-7.

Campanello, P. I., P. Mac Donagh, and G. Goldstein. 2009. Reduced-impact logging and post-harvest mamagement in the atlantic forest of Argentina: alternative approaches to enhance regeneration and growth of canopy trees. Pp. 39-59 in S. P. Rossberg (ed.). Forest Management. Nova Science, New York, USA.

Campanello, P. I., J. F. Garibaldi, M. G. Gatti, and G. Goldstein. 2007a. Lianas in a subtropical Atlantic Forest: Host preference and tree growth. Forest Ecology and Management 242:250-259. https://doi.org/10.1016/j.foreco.2007.01.040. 
Campanello, P. I., M. Genoveva Gatti, A. Ares, L. Montti, and G. Goldstein. 2007b. Tree regeneration and microclimate in a liana and bamboo-dominated semideciduous Atlantic Forest. Forest Ecology and Management 252:108-117. https: //doi.org/10.1016/j.foreco.2007.06.032.

Chuine, I., and E. G. Beaubien. 2001. Phenology is a major determinant of tree species range. Ecology Letters 4:500-510. https://doi.org/10.1046/j.1461-0248.2001.00261.x.

Chuine, I., and J. Régnière. 2017. Process-Based Models of Phenology for Plants and Animals. Annual Review of Ecology, Evolution, and Systematics 48:159-182. https://doi.org/10.1146/annurev-ecolsys-110316-022706.

Cristiano, P., N. Madanes, P. Campanello, D. di Francescantonio, S. Rodríguez, Y.-J. Zhang, L. Carrasco, and G. Goldstein. 2014. High NDVI and Potential Canopy Photosynthesis of South American Subtropical Forests despite Seasonal Changes in Leaf Area Index and Air Temperature. Forests 5:287-308. https://doi.org/10.3390/f5020287.

Davies, T. J., E. M. Wolkovich, N. J. B. Kraft, N. Salamin, A. M., T. R. Ault, J. L. Betancourt, K. Bolmgren, E. E. Cleland, B. I. Cook, and T. M. Crimmins. 2013. Phylogenetic conservatism in plant phenology. Journal of Ecology 101:15201530. https://doi.org/10.1111/1365-2745.12154.

Denny, E. G., K. L. Gerst, A. J. Miller-Rushing, G. L. Tierney, T. M. Crimmins, C. A. F. Enquist, P. Guertin, A. H. Rosemartin, M. D. Schwartz, K. A. Thomas, and J. F. Weltzin. 2014. Standardized phenology monitoring methods to track plant and animal activity for science and resource management applications. International Journal of Biometeorology 58:591-601. https://doi.org/10.1007/s00484-014-0789-5.

Deslauriers, A., S. Rossi, and T. Anfodillo. 2007. Dendrometer and intra-annual tree growthø: What kind of information can be inferred? Dendrochronologia 25:113-124. https://doi.org/10.1016/j.dendro.2007.05.003.

Forrest, J., and A. J. Miller-Rushing. 2010. Toward a synthetic understanding of the role of phenology in ecology and evolution. Philosophical Transactions of the Royal Society B: Biological Sciences 365:3101-3112. https://doi.org/ 10.1098/rstb.2010.0145.

di Francescantonio, D., M. Villagra, G. Goldstein, and P. I. Campanello. 2018. Leaf phenology and water-use patterns of canopy trees in Northern Argentinean subtropical forests. Tree Physiology 38:1841-1854. https://doi.org/10.1093/ treephys/tpy072.

Franco, A. M. S. 2008. Estrutura, diversidade e aspectos ecológicos do componente arbustivo e arbóreo em uma floresta estacional, Parque Estadual do Turvo, sul do Brasil. Universidad Federal do Rio Grande Do Sul.

Fu, Y. H., X. Zhang, S. Piao, F. Hao, X. Geng, Y. Vitasse, C. Zohner, J. Peñuelas, and I. A. Janssens. 2019. Daylength helps temperate deciduous trees to leaf-out at the optimal time. Global Change Biology 25:2410-2418. https://doi.org/ $10.1111 / \mathrm{gcb} .14633$

Gatti, M. G., P. I. Campanello, L. F. Montti, and G. Goldstein. 2008. Frost resistance in the tropical palm Euterpe edulis and its pattern of distribution in the Atlantic Forest of Argentina. Forest Ecology and Management 256:633-640. https: //doi.org/10.1016/j.foreco.2008.05.012.

Hijmans, R. J. 2016. geosphere: spherical trigonometry R package. URL: https://tinyurl.com/ydhhw6sn.

Hódar, J. A., R. Zamora, and J. Peñuelas. 2004. El efecto del Cambio Global en las interacciones planta- animal. Pp. 461-478 in F. Valladares (ed.). Ecología del bosque mediterráneo en un mundo cambiante. Madrid.

IBGE. 2012. Manual Técnico da Vegetação Brasileira. Série Manuais Técnicos em Geociências 1. 2nd edition. IBGEInstituto Brasileiro de Geografia e Estatística, Rio de Janeiro, Brasil.

Jardim Botânico do Rio de Janeiro. 2018. Flora do Brasil 2020. Under construction.

Kikuzawa, K., and M. J. Lechowicz. 2011. Ecology of Leaf Longevity. Springer Tokyo, Tokyo. https://doi.org/10.1007/ 978-4-431-53918-6.

Kikuzawa, K., Y. Onoda, I. J. Wright, and P. B. Reich. 2013. Mechanisms underlying global temperature-related patterns in leaf longevity. Global Ecology and Biogeography 22:982-993. https://doi.org/10.1111/geb.12042.

Köcher, P., V. Horna, and C. Leuschner. 2013. Stem water storage in five coexisting temperate broad-leaved tree species: Significance, temporal dynamics and dependence on tree functional traits. Tree Physiology 33:817-832. https: //doi.org/10.1093/treephys/tpt055.

Kramer, K., B. Degen, J. Buschbom, T. Hickler, W. Thuiller, M. T. Sykes, and W. de Winter. 2010. Modelling exploration of the future of European beech (Fagus sylvatica L.) under climate change-Range, abundance, genetic diversity and adaptive response. Forest Ecology and Management 259:2213-2222. https://doi.org/10.1016/j.foreco.2009.12.023.

Lasky, J. R., M. Uriarte, and R. Muscarella. 2016. Synchrony, compensatory dynamics, and the functional trait basis of phenological diversity in a tropical dry forest tree community: effects of rainfall seasonality. Environmental Research Letters 11:115003. https://doi.org/10.1088/1748-9326/11/11/115003.

Lund, U., C. Agostinelli, and M. C. Agostinelli. 2017. Package 'circular.' CRAN Repository.

Marques, M. C. M., J. J. Roper, and A. P. Baggio Salvalaggio. 2004. Phenological patterns among plant life-forms in a subtropical forest in southern Brazil. Plant Ecology 173:203-213. https://doi.org/10.1023/B:VEGE.0000029325.85031 .90 .

Morellato, L. P. C., B. Alberton, S. T. Alvarado, B. Borges, E. Buisson, M. G. G. Camargo, L. F. Cancian, D. W. Carstensen, D. F. E. Escobar, P. T. P. Leite, I. Mendoza, N. M. W. B. Rocha, N. C. Soares, T. S. F. Silva, V. G. Staggemeier, A. S. Streher, B. C. Vargas, and C. A. Peres. 2016. Linking plant phenology to conservation biology. Biological Conservation 195:60-72. https://doi.org/10.1016/j.biocon.2015.12.033.

Morellato, P., L. F. Alberti, and I. Hudson. 2010. Applications of Circular Statistics in Plant Phenology: a Case Studies Approach. Pages 339-359 in I. L. Hudson and M. Keatley (eds.). Phenological Research: Methods for Environmental and Climate Change Analysis. Springer, Dordrecht. https://doi.org/10.1007/978-90-481-3335-2_16. 
Morellato, P., D. Talora, A. Takahasi, C. Bencke, E. Romera, and V. Zipparro. 2000. Phenology of Atlantic Rain Forest Trees: A Comparative Study 1. Biotropica 32:811-823. https://doi.org/10.1646/0006-3606(2000)032[0811:POARFT]2.0.CO;2. https://doi.org/10.1111/j.1744-7429.2000.tb00620.x.

Oliveira-Filho, A. T., J. C. Budke, J. A. Jarenkow, P. V. Eisenlohr, and D. R. M. Neves. 2015. Delving into the variations in tree species composition and richness across South American subtropical Atlantic and Pampean forests. Journal of Plant Ecology 8:242-260. https://doi.org/10.1093/jpe/rtt058.

Oliveira-Filho, A. T., and M. A. Fontes. 2000. Patterns of Floristic Differentiation among Atlantic Forests in Southeastern Brazil and the Influence of Climate. Biotropica 32:793-810. https://doi.org/10.1646/0006-3606(2000)032[0793: POFDAA]2.0.CO;2. https://doi.org/10.1111/j.1744-7429.2000.tb00619.x.

Osada, N., and T. Hiura. 2019. Intraspecific differences in spring leaf phenology in relation to tree size in temperate deciduous trees. Tree Physiology 39:782-791. https://doi.org/10.1093/treephys/tpz011.

Oyazabal, M., J. Clavijo, L. Oakley, F. Biganzoli, P. Tognetti, I. Barberis, H. Maturo, R. Aragón, P. I. Campanello, D. Prado, M. Oesterheld, and R. J. C. León. 2018. Unidades de vegetación de la Argentina. Ecologia Austral 28:40-63. https://doi.org/10.25260/EA.18.28.1.0.399.

Panchen, Z. A., R. B. Primack, B. Nordt, E. R. Ellwood, A. D. Stevens, S. S. Renner, C. G. Willis, R. Fahey, A. Whittemore, Y. Du, and C. C. Davis. 2014. Leaf out times of temperate woody plants are related to phylogeny, deciduousness, growth habit and wood anatomy. New Phytologist 203:1208-1219. https://doi.org/10.1111/nph.12892.

Pennington, R. T., M. Lavin, and A. Oliveira. 2009. Woody Plant Diversity, Evolution, and Ecology in the Tropics: Perspectives from Seasonally Dry Tropical Forests. Annual Review of Ecology Evolution and Systematics 40:437-457. https://doi.org/10.1146/annurev.ecolsys.110308.120327.

Pewsey, A., M. Neuhauser, and G. Ruxton. 2013. Circular statistics in R. Oxford University Press, Oxford, United Kingdom.

Di Rienzo, J. A., F. Casanoves, M. G. Balzarini, L. Gonzalez, M. Tablada, and C. W. Robledo. 2017. InfoStat versión (2017). Grupo InfoStat, FCA, Universidad Nacional de Córdoba, Argentina.

Rossi, S., and J. Bousquet. 2014. The bud break process and its variation among local populations of boreal black spruce. Frontiers in Plant Science 5:1-9. https://doi.org/10.3389/fpls.2014.00574.

van Schaik, C. P., J. W. Terborgh, and S. J. Wright. 1993. The Phenology of Tropical Forests: Adaptive Significance and Consequences for Primary Consumers. Annual Review of Ecology and Systematics 24:353-377. https://doi.org/ 10.1146/annurev.es.24.110193.002033.

Seki, M., T. Yoshida, and T. Takada. 2015. A general method for calculating the optimal leaf longevity from the viewpoint of carbon economy. Journal of Mathematical Biology 71:669-690. https://doi.org/10.1007/s00285-014-0830-7.

Shimamoto, C. Y., P. C. Botosso, E. Amano, and M. C. M. Marques. 2016. Stem growth rhythms in trees of a tropical rainforest in Southern Brazil. Trees-Structure and Function 30:99-111. https://doi.org/10.1007/s00468-015-1279-z.

Singh, R. K., T. Svystun, B. AlDahmash, A. M. Jönsson, and R. P. Bhalerao. 2017. Photoperiod- and temperaturemediated control of phenology in trees - a molecular perspective. New Phytologist 213:511-524. https://doi.org/ 10.1111/nph.14346.

Srur, M., F. Gatti, V. Benesovsky, J. Herrera, R. Melzew, and M. Camposano. 2007. Identificación, caracterización y mapeo de los ambientes del Parque Nacional Iguazú. Puerto Iguazú.

Veloso, H. P., A. L. R. Rangel-Filho, and J. C. A. Lima. 1991. Classificação da vegetação brasileira, adaptada a um sistema universal. Page (I. B. de G. e Estatística, Ed.). Rio de Janeiro, Brasil.

Vitasse, Y. 2013. Ontogenic changes rather than difference in temperature cause understory trees to leaf out earlier. New Phytologist 198:149-155.

Vitasse, Y., A. Lenz, and C. Körner. 2014. The interaction between freezing tolerance and phenology in temperate deciduous trees. Frontiers in Plant Science 5:1-12. https://doi.org/10.1111/nph.12130.

Werneck, F. P. 2011. The diversification of eastern South American open vegetation biomes: Historical biogeography and perspectives. Quaternary Science Reviews 30:1630-1648. https://doi.org/10.1016/j.quascirev.2011.03.009.

Zar, J. H. 1999. Biostatistical Analysis. Prentice-Hall Inc., New Jersey.

Zhang, Y.-J., P. M. Cristiano, Y.-P. Zhang, K.-F. Cao, and G. H. Goldstein. 2016. Carbon Economy of Subtropical Forests. Page in G. Goldstein and L. S. Santiago (eds.). Tropical Tree Physiology. Springer. https://doi.org/10.1007/978-3-31927422-5_16.

Zuloaga, F., O. Morrone, and M. Belgrano. 2008. Catálogo de plantas vasculares del Cono Sur. Missouri Botanical Garden Press. 